\title{
DOCK8 immune deficiency as a model for primary cytoskeletal dysfunction
}

\author{
Sean A. McGhee and Talal A. Chatila* \\ Department of Pediatrics, David Geffen School of Medicine at UCLA, Los Angeles, CA, USA
}

\begin{abstract}
DOCK8 deficiency is a newly described primary immune deficiency resulting in profound susceptibility to cutaneous viral infections, elevated IgE levels, and eosinophilia, but lacking in the skeletal manifestations commonly seen in hyper IgE syndrome, which it otherwise resembles. Although little is known about the DOCK8 protein, it resembles other atypical guanine exchange factors in the DOCK family, and is known to bind to CDC42. This suggests that a likely role for DOCK8 is in modulating signals that trigger cytoskeletal reorganization. As a result, DOCK8 may also be related to other immune deficiencies that involve the cytoskeleton and Rho GTPase signaling pathways, such as Wiskott-Aldrich syndrome and Rac2 deficiency.
\end{abstract}

Keywords: DOCK8, cytoskeleton, immune deficiency

\section{Introduction}

In 2009, DOCK8 (Dedicator of cytokinesis 8) was independently determined to be the cause of significant immune deficiency both in humans and in mice [1-3]. These discoveries were made from positional data in the case of the human disease [1,2], and from a mutagenesis screen in the case of the mouse disease [3]. Little prior information was available as to the protein or its function. Indeed, most of the current understanding of the protein is based on argument by analogy to other proteins within the same family and with similar domain structures. If these analogies hold true, however, DOCK8 has the potential to further elucidate a relatively poorly understood mechanism for generating immune deficiency, that of disorders of cytoskeletal function.

\subsection{DOCK8 deficiency as a cytoskeletal disorder involving Rho GTPases}

Cytoskeletal dysfunction as a cause of immune deficiency is currently best understood in the setting of

*Corresponding author: Talal A. Chatila, MD, Division of Pediatric Immunology, MDCC 12-430, 10833 Le Conte Ave, Los Angeles, CA 90095-1752, USA. Tel.: +1 310825 6481; E-mail: tchatila@mednet.ucla.edu. the Wiskott-Aldrich syndrome (WAS), a defect in an initiator of cytoskeletal remodeling and branching that results in a syndrome of progressive immune deficiency, with rash, elevated IgE, and a susceptibility to autoimmunity [4]. Many of these features are also present in DOCK8 deficiency, and the general structure of DOCK8 suggests that it may be involved in the same Rho GTPase signaling pathway as WAS [5].

This idea is primarily drawn from the general domain structure of DOCK8 and the protein family with which it is classified. DOCK8 is an atypical guanine exchange factor (GEF). GEFs, in general, have responsibility for modulating the activity of Rho GTPases, which in turn have a key role in integrating signals from the cell membrane and triggering appropriate cytoskeletal reorganization as a result [6]. This integration follows a model in which the activity of the Rho GTPase is a simple switch, active when bound to GTP and inactive when bound to GDP. Whether the GTPase is GDP or GTP bound is determined by the activity of at least two classes of proteins: GEFs, which cycle the GTPase to activate it, and GTPase activating proteins (GAPs), which cycle GTPases back to the inactive state (Fig. 1). There are at least 20 Rho GTPases known, the best known of which are CDC42, Rac1, Rac2, RhoA, RhoB, and RhoH [7]. Each interacts with specific GEFs 


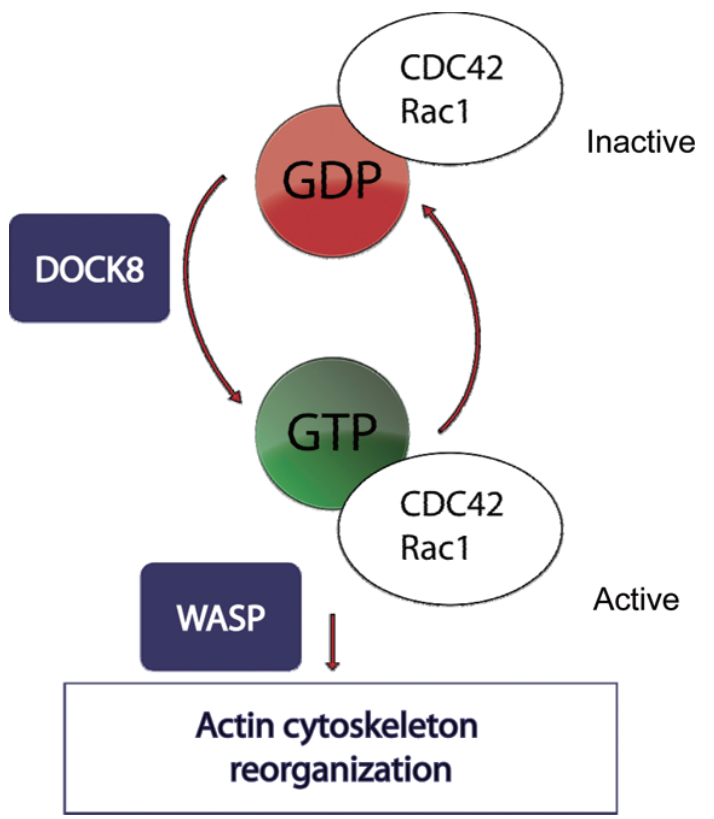

Fig. 1. Putative role of DOCK8 in transduction of signals through CDC42 and Rac to the cytoskeleton. WASp acts as a downstream effector for signals from Rho GTPases such as CDC42 and Rac. These GTPases are, in turn, regulated by the activity of guanine exchange factors, such as DOCK8, which cycle the GTPase from inactive to active form.

and GAPs, although there is considerable redundancy and overlap in the specificity of these interactions.

The GEFs were originally described as a group of about 70 proteins that were structurally related to the diffuse B cell lymphoma protein ( $\mathrm{dbl})$ in containing a pleckstrin homology domain (PH) and a dbl homology (DH) domain with GEF activity. The DOCK proteins, in contrast, have GEF activity but have a different domain structure [6].

\subsection{DOCK protein structure and function}

DOCK proteins do not have DH domains, but rather two dock homology domains, DHR1 and DHR2. Of the 11 known DOCK proteins, the family of which DOCK8 is a member, the first and best known is DOCK180. For DOCK180, the DHR1 domain binds phosphotidylinositol trisphosphate, while the DHR2 domain interacts with Rho GTPases, and most of the other DOCK proteins appear to follow this model [8,9]. However, DOCK proteins also appear to have other interactions, including with adaptor proteins at the cell membrane (CrkII and ELMO, in the case of DOCK180), and DOCK9 has been shown to oligomerize through its DHR2 domain [6]. Hence, DOCK function could in- volve the formation of oligomers and interactions with a wide range of proteins. In addition, DOCKs have putative phosphorylation sites suggesting additional potential levels of regulation $[6,10]$. In the case of DOCK8, this is a single phosphoserine at position 451 .

To date, potential interactions between DOCK8 and several GTPases have been demonstrated, including Cdc42, Rac1, RHOJ, RHOQ [5]. These were demonstrated in a yeast two-hybrid system, but not confirmed when the results were repeated in a GST pull-down with any of a number of Rho GTPases. While it was speculated that this was due to unstable binding of DOCK8 to the Rho GTPases, clear binding partners for DOCK8 remain undefined.

DOCK8 protein is present in subsets of wide range of cells, and the mRNA is widely distributed throughout most tissues [5]. Staining for the protein in histological sections does reveal some predilection for certain cell types [11]. As might be expected given the phenotype of immune deficiency, there is strong expression in hematopoietic cells and normal peripheral blood mononuclear cells (PBMCs). Within the lungs, there is strong expression in lung macrophages. While most cells in germinal centers have strong expression of DOCK8, it is not evenly expressed throughout the germinal center.

Glandular tissue, such as adrenal and prostate, endometrium and trophoblast appear to express the protein, and it is present in some neural tissues, though not in the cerebellum or cerebral cortex. Leukemia cells express DOCK8 in a variety of subtypes at high levels compared to other cell lines, but, of course, so do normal peripheral blood mononuclear cells [11]. Changes in the expression pattern of DOCK8 have also been identified in a variety of different tumors. These changes have included both decreases $[12,13]$ and increases [14] in DOCK8 expression as well as deletions of genomic copies of DOCK8 [12,13,15].

Also interesting is the subcellular localization of DOCK 8 by immunofluorescence. In cell lines, the protein is diffusely distributed throughout the cytoplasm in a distinctly granular pattern, suggesting that there may be significant interactions with other proteins or the cytoskeleton [6,11]. The amount of staining appears to intensify in lamellipodia, but the granular distribution persists elsewhere. Other GEFs and Rho GTPases tend to localize at the cell membranes [16,17]. Furthermore, there is DOCK8 staining in the nucleus, exempting nucleolar regions. All of these staining studies to date have been done in cell lines, though, and so may differ from normal expression patterns for the protein, and 
the question has not been studied in sufficient detail to know whether this is a pre- or post-activation pattern.

Most Rho GTPases cluster at the cell membrane, receive signals from cell signaling molecules such as integrins, and transduce those signals to the cytoskeleton. Rho GTPases modulate cytoskeletal function using a model in which the Rho GTPase acts to integrate the function of cell surface signaling molecules, and passes this signal to an effector molecule that develops the reorganization of the cytoskeleton [7]. The integration of the signal by the Rho GTPase is modified by activating and inhibiting proteins that modulate whether the signal is passed to the effector. These include the GEFs, as well as GAPs, which switch the GTP for GDP and so disable the Rho GTPase. This step is where DOCK8 modulates cytoskeletal function. The effect on the cytoskeleton is in reorganization and concentration of Factin filaments in specific subcellular areas. For immune system cells, this includes modulating the formation of podosomes, and formation of the immunological synapse. Other functions of Rho GTPases include activation of NF- $\kappa \mathrm{B}$, which could also have an effect on immune function [18].

There are many cellular processes, particularly in hematopoietic and immune tissues, that Rho GTPases regulate, and so would be candidates for modulation by DOCK8 [7]. Rho GTPases receive signals from integrins in hematopoietic stem cells and have roles in homing, engraftment, and mobilization. As expected, the Rho GTPase is primarily involved in the cytoskeletal reorganization that is required for these processes. In T cells, Rho GTPases mediate polarity, cytotoxic response, spreading, migration, cytokine secretion, TCR signaling and in so doing, regulate key transitions in thymocyte development $[19,20]$. In mice, if Rac1 and Rac2 are both deleted, T-cell development is blocked at the common lymphoid progenitor stage, there is disruption of actin polymerization and TCR clustering, and no calcium influx, Erk, or p38 activation [7,21]. In B cells, Rac2 deficient mice have reduction in $\mathrm{B} 1 \mathrm{a}$ and MZ B cells, associated with impaired proliferation and calcium influx, as do double knockouts of Rac1 and Rac2. B cells with Cdc42 deletions are similar to these Rac deficient mice [22]. Some inhibitory Rho GTPases also exist, and RhoH inhibits the Rac1 and Rho activation of NF- $\kappa \mathrm{B}$ and is known to bind to Zap-70.

The Rho GTPase pathway uses the Rho GTPase as an adjustable switch for passing signals to an effector molecule. The best understood effector for the Rho GTPase pathway is the WASp (Wiskott-Aldrich syndrome protein), which causes a syndrome of immune deficiency with rash, elevated $\mathrm{IgE}$, and defective immune synapse very similar in some ways to DOCK8 deficiency. As an effector for CDC42 and Rac, WASp initiates the branching of F-actin filaments via activation of the Arp2/3 complex [4]. Therefore, we now have deficiencies of all three major components of Rho GTPase signaling pathway causing immune defects, including the effectors, such as WASp, the Rho GTPase itself, such as in dominant negative Rac2 deficiency, and the modulators, such as DOCK8.

\subsection{DOCK8 deficiency in humans}

The syndrome ultimately identified as DOCK8 deficiency in humans was originally described as an autosomal recessive form of hyper IgE syndrome (AR-HIES), with which it shares several features (Table 1) [23]. These include elevated IgE and eosinophilia, susceptibility to cutaneous infections, and a defect in TH17 cell development and function. However, viral infections were much more prominent in DOCK8 patients, and these patients had a greater susceptibility to autoimmune disease than hyper-IgE syndrome patients. Initial searches for mutations in candidate genes within the STAT3 pathway, which is disrupted in autosomal dominant hyper-IgE syndrome, were not successful. However, when high-density oligonucleotide mapping arrays were used to assess gene copy number, homozygous deletions of the DOCK8 gene were found in many families with AR-HIES, and mutations resulting in stop codons and exon skipping were found in DOCK8 in many of the remaining families [1,2]. Therefore, DOCK8 was identified as the causative gene for AR-HIES based solely on positional data.

\subsection{DOCK8 in non-human model systems}

At approximately the same time that the human syndrome was discovered, a mouse immunization genetic screen identified the mouse homolog of DOCK8, Dock8, as a candidate for poor immunization responses. This mutant mouse was discovered in an ENU mutagenesis screen in which the mutant mice failed to sustain a primary antibody response and did not develop mature antibody responses through somatic hypermutation [3]. Two separate mutations in the same gene were discovered in different lines, one resulting in a splice donor mutation that eliminated the DHR2 domain, and another in the alpha helix responsible for CDC42 binding in the DHR2 domain. In these mice, the early phase of antibody production was indistin- 
Table 1

Phenotypic differences between patients with autosomal dominant HIES (STAT3 deficient) and autosomal recessive HIES (DOCK8 deficient)

\begin{tabular}{lcc}
\hline & $\begin{array}{c}\text { STAT3 deficient } \\
\text { HIES }\end{array}$ & $\begin{array}{c}\text { STAT3 normal HIES } \\
\text { (DOCK8 deficient) }\end{array}$ \\
\hline Inheritance & Autosomal Dominant & Autosomal Recessive \\
Respiratory Infections & + & + \\
Elevated IgE & + & + \\
Pneumatoceles & Characteristic & Rare \\
Cutaneous viral infections & Present & Marked \\
Fractures & + & - \\
Primary Dentition & Retained & Not retained \\
Autoimmunity & Possible & Frequent \\
Facial features & + & $+/-$ \\
\hline
\end{tabular}

guishable from normal mice, but the later phases were diminished, antibody production did not persist, and the mice did not undergo hypermutation and affinity maturation.

There was no effect on $\mathrm{T}$ independent antigen, suggesting that a disorder of the $\mathrm{T}$ and $\mathrm{B}$ cell immune synapse could be important [3]. T and B cell numbers were generally normal, but there was a clear deficiency of marginal zone B cells and reduced numbers of naive $\mathrm{T}$ cells. Further study demonstrated a defective B-cell synapse and abnormal function of cytoskeletal processes. In normal B cells, recognition of antigen causes binding of LFA-1 to ICAM-1, resulting in $\mathrm{B}$ cell adhesion and spreading. The supramolecular activation complex (SMAC) is formed, and LFA becomes polarized in peripheral SMAC. In Dock8 deficient mice, the mutant cells were unable to appropriately cluster ICAM-1 into peripheral SMAC. Rho GTPases are known to be involved in integrating the signals from integrins, further suggesting that a defect in the Rho GTPase pathway may be important in this syndrome. There was no effect on other BCR signaling events or chemotaxis.

One of the dilemmas in the descriptions of the mouse disease and the human disease is that the clinical picture appears to vary between the two. This may be partly due to the manner in which the mouse was discovered; as part of a mutagenesis screen for antibody deficiency the mouse was primarily ascertained for antibody defects, whereas resistance to viral cutaneous infections or susceptibility to autoimmunity was not assessed. While humans with DOCK8 deficiency do manifest variable antibody defects, it remains to be established whether some DOCK8 mutations may manifest primarily as common variable immune deficiency.

Although the difference in study design may explain part of this, there may also be genuine differences between the mouse model and the human disease. This is true for the best understood disease of the Rho GTPase pathway, Wiskott-Aldrich syndrome. While mice carrying WASp mutations similar to human patients do replicate many of the laboratory features of WAS, they do not have an obvious clinical phenotype [24,25]. The reasons for these differences between mice and humans are not completely clear, but given that many other proteins and systems appear to modify the function of Rho GTPase signaling, it may well be that these systems play somewhat different roles in the immune systems of mice and humans.

Another important dilemma is how such a broad defect in cytoskeletal signaling is manifested in a relatively limited phenotype, immune deficiency. Patients with DOCK8 deficiency do not have the same skeletal findings, such as fractures, scoliosis and retention of primary dentition, which subjects with STAT3 deficiency do $[2,23,26]$. There may be some developmental or cognitive delay, but this has not been formally ascertained. Other patients with single copy deletions of DOCK8 have shown cognitive delay, but this is not necessarily the case in all the patients seen with loss of both copies of DOCK8 [27,28]. A nearby gene, ANKRD15, has been associated with familial cerebral palsy and developmental delay [29], and it is possible that this gene, and not DOCK8, is responsible for the neurologic findings in at least one of these patients [27], as the deletion did involve ANKRD15. However, the other patient had a deletion that did not involve ANKRD15 and disrupted DOCK8 [28]. Part of this may be explained by restriction of DOCK8 expression to immune tissues, but DOCK8 is also found in glandular tissue, heart, and skeletal muscle, and defects in these systems are not evident. This suggests that the role of DOCK8 might be redundant or less important in these other tissues.

As the primary effector of CDC42 signaling, WASp function may also be diminished in DOCK8 patients, if CDC42 lacks signal from DOCK8 to upregulate its 
function. Although the entire phenotype of DOCK8 deficiency cannot be explained by WASp deficiency alone, many laboratory features of WASp deficiency that have been tested in DOCK8 deficiency are also replicated, at least in the mouse. WASp deficiency results in abnormal adherence to ICAM-1 coated surfaces, in a poor development of the immune synapse, and WASp's activation of Arp2/3 to produce actin reorganization is enhanced by CDC42, known to be a binding partner of DOCK8. Furthermore, the clinical appearance of WAS deficient humans is similar to DOCK8 deficiency. WASp deficient patients are susceptible to viral, pyogenic, and opportunistic infections and have defective CD3- and mitogen-induced proliferation of T cells, as do DOCK8 deficient patients. WAS patients also have elevated IgE levels with time, and an eczematous rash. There is also a progressive decrease in number and function of T cells [30]. It is currently unclear how a fixed defect in CDC42 signaling would lead to a progressive immune deficiency. The features of DOCK8 deficiency that cannot be explained by WASp deficiency, such as the marked presence of cutaneous viral infections, might be explained by interactions between DOCK8 and other Rho GTPases, or possibly DOCK8's influence on other signaling pathways altogether.

Other defects in Rho GTPase signaling besides WAS also seem to share features with DOCK8 deficiency. The clinical phenotype is similar to defects in Rac2, and Vav1 or Vav2, although these may be more restricted to B cell function in mice $[3,31]$. DOCK2 deficiency, identified so far only in mice, is similar, but more profound than the DOCK8 picture, with lymphopenia, disrupted lymphoid architecture, and poor B cell migration and chemotaxis $[32,33]$.

To date, much of this argument for the function of DOCK8 in producing immune deficiency is made by analogy from the few known binding partners for the protein and the general structure of the protein. It will be critical to outline in much greater detail the interactions and distribution of DOCK8 both throughout tissues and subcellularly. This will be key in both mice and humans to determine what, if any, differences truly exist between the mouse model and the human disease, and also to determine why the phenotype is largely restricted to immunologic function.

Not only will this be important for better understanding pathogenesis and perhaps treatment of those with DOCK8 deficiency, but the cytoskeleton and cytoskeletal function is an underappreciated site for intervention to modify immune function pharmacologically. Both colchicine and hydroxychloroquine have found use as clinical agents for controlling undesirable inflammation, and both appear to have effects on cytoskeletal processes, such as microtubule polymerization and vesicle transport, respectively. The cytoskeleton appears susceptible to modification by small molecule agents, which would improve the options for drug delivery over traditional large molecule agents like monoclonal antibodies. A better appreciation of the mechanisms and interactions that are involved in the production of cytoskeletal immune defects could clear the path for antiinflammatory agents that are better tolerated, easier to administer and have less susceptibility to broad, unwanted effects.

\section{References}

[1] K.R. Engelhardt, S. McGhee, S. Winkler, A. Sassi, C. Woellner, G. Lopez-Herrera et al., Large deletions and point mutations involving the dedicator of cytokinesis 8 (DOCK8) in the autosomal-recessive form of hyper-IgE syndrome, J Allergy Clin Immunol 124 (2009), 1289-1302.

[2] Q. Zhang, J.C. Davis, I.T. Lamborn, A.F. Freeman, H. Jing, A.J. Favreau et al., Combined Immunodeficiency associated with DOCK8 mutations, N Engl J Med 361 (2009), 20462055.

[3] K.L. Randall, T. Lambe, A. Johnson, B. Treanor, E. Kucharska, H. Domaschenz et al., Dock8 mutations cripple B cell immunological synapses, germinal centers and long-lived antibody production, Nat Immunol 10 (Dec 2009), 1283-1291.

[4] A.J. Thrasher and S.O. Burns, WASP: a key immunological multitasker, Nat Rev Immunol 10 (Mar 2010), 182-192.

[5] A. Ruusala and P. Aspenström, Isolation and characterization of DOCK8, a member of the DOCK180-related regulators of cell morphology, FEBS Lett 572 (2004), 159-166.

[6] Y. Miyamoto and J. Yamauchi, Cellular signaling of Dock family proteins in neural function, Cell Signal 22 (Feb 2010), 175-182.

[7] J.C. Mulloy, J.A. Cancelas, M.D. Filippi, T.A. Kalfa, F. Guo and Y. Zheng, Rho GTPases in hematopoiesis and hemopathies, Blood 115 (4 Feb 2010), 936-947.

[8] J.F. Côté and K. Vuori, GEF what? Dock180 and related proteins help Rac to polarize cells in new ways, Trends Cell Biol 17 (Aug 2007), 383-393.

[9] N. Meller, S. Merlot and C. Guda, CZH proteins: a new family of Rho-GEFs, J Cell Sci 118 (1 Nov 2005), 4937-4946.

[10] F.S. Oppermann, F. Gnad, J.V. Osen, R. Hornberger, Z. Greff, G. Keri et al., Large-scale proteomics ianalysis of the human kinome, Mol Cell Proteomics 8 (2009), 1751-1764.

[11] L. Berglund et al., A gene-centric human protein atlas for expression profiles based on antibodies, Mol Cell Proteomics 10 (2008), 2019-2027.

[12] P. Saelee, S. Wongkham, A. Puapairoj, N. Khuntikeo, S. Petmitr, S. Chariyalertsak et al., Novel PNLIPRP3 and DOCK8 gene expression and prognostic implications of DNA loss on chromosome 10q25.3 in hepatocellular carcinoma, Asian Pac J Cancer Prev 10 (Jul-Sep 2009), 501-506.

[13] K. Takahashi, T. Kohno, R. Ajima, H. Sasaki, J.D. Minna, T. Fujiwara et al., Homozygous deletion and reduced expression 
of the DOCK8 gene in human lung cancer, Int J Oncol $\mathbf{2 8}$ (Feb 2006), 321-328.

[14] R. Ogawa, H. Ishiguro, Y. Kuwabara, M. Kimura, A. Mitsui, Y. Mori et al., Identification of candidate genes involved in the radiosensitivity of esophageal cancer cells by microarray analysis, Dis Esophagus 21 (2008), 288-297.

[15] A. Idbaih, R. Carvalho Silva, E. Crinière, Y. Marie, C. Carpentier, B. Boisselier et al., Genomic changes in progression of low-grade gliomas, J Neurooncol 90 (Nov 2008), 133-140.

[16] A. Schimdt and A. Hall, Guanine nucleotide exchange factors for Rho GTPases: turning on the switch, Genes Dev 16 (2002), 1587-1609.

[17] D. Michaelson, J. Silletti, G. Murphy, P. D’Eustachio, M. Rush and M.R. Philips, Differential localization of Rho GTPases in live cells: regulation by hypervariable regions and RhoGDI binding, J Cell Biol 152 (8 Jan 2001), 111-126.

[18] R. Perona, S. Montaner, L. Saniger, I. Sánchez-Pérez, R. Bravo and J.C. Lacal, Activation of the nuclear factor-kappaB by Rho, CDC42, and Rac-1 proteins, Genes Dev 11 (15 Feb 1997), 463-475.

[19] M. Gomez, V. Tybulewicz and D.A. Cantrell, Control of pre-T cell proliferation and differentiation by the GTPase Rac-I, Nat Immunol 1 (Oct 2000), 348-352.

[20] P. Lorès, L. Morin, R. Luna and G. Gacon, Enhanced apoptosis in the thymus of transgenic mice expressing constitutively activated forms of human Rac2GTPase, Oncogene 15 (31 Jul 1997), 601-605.

[21] F. Guo, J.A. Cancelas, D. Hildeman, D.A. Williams and Y. Zheng, Rac GTPase isoforms Rac1 and Rac2 play a redundant and crucial role in T-cell development, Blood 112(5) (1 Sep 2008), 1767-1775

[22] F. Guo, C.S. Velu, H.L. Grimes and Y. Zheng, Rho GTPase Cdc42 is essential for B-lymphocyte development and activation, Blood 114 (1 Oct 2009), 2909-2916. Epub 11 Aug 2009.

[23] E.D. Renner, J.M. Puck, S.M. Holland, M. Schmitt, M. Weiss, M. Frosch et al., Autosomal recessive hyperimmunoglobulin E syndrome: a distinct disease entity, J Pediatr 144 (2004), 93-99.

[24] S.B. Snapper, F.S. Rosen, E. Mizoguchi, P. Cohen, W. Khan,
C.H. Liu et al., Wiskott-Aldrich syndrome protein-deficient mice reveal a role for WASP in T but not B cell activation, Immunity 9 (Jul 1998), 81-91.

[25] J. Zhang, A. Shehabeldin, L.A. da Cruz, J. Butler, A.K. Somani, M. McGavin et al., Antigen receptor-induced activation and cytoskeletal rearrangement are impaired in WiskottAldrich syndrome protein-deficient lymphocytes, J Exp Med 190 (1 Nov 1999), 1329-1342.

[26] S. Al Khatib, S. Keles, M. Garcia-Lloret, E. Karakoc-Aydiner, I. Reisli, $\mathrm{H}$. Artac et al., Defects along the $\mathrm{T}_{\mathrm{H}} 17$ differentiation pathway underlie genetically distinct forms of the hyper $\operatorname{IgE}$ syndrome, J Allergy Clin Immunol 124 (2009), 342-348.

[27] G. Vinci, S. Chantot-Bastaraud, B. El Houate, S. Lortat-Jacob, R. Brauner and K. McElreavey, Association of deletion 9p, 46,XY gonadal dysgenesis and autistic spectrum disorder, $\mathrm{Mol}$ Hum Reprod 13 (Sep 2007), 685-689.

[28] B.L. Griggs, S. Ladd, R.A. Saul, B.R. DuPont and A.K. Srivastava, Dedicator of cytokinesis 8 is disrupted in two patients with mental retardation and developmental disabilities, Genomics 91 (Feb 2008), 195-202.

[29] I. Lerer, M. Sagi, V. Meiner, T. Cohen, J. Zlotogora and D. Abeliovich, Deletion of the ANKRD15 gene at 9p24.3 causes parent-of-origin-dependent inheritance of familial cerebral palsy, Hum Mol Genet 14 (15 Dec 2005), 3911-3920.

[30] A.J. Thrasher, WASp in immune-system organization and function, Nat Rev Immunol 2(9) (Sep 2002), 635-646.

[31] E. Arana, A. Vehlow, N.E. Harwood, E. Vigorito, R. Henderson, M. Turner et al., Activation of the small GTPase Rac2 via the B cell receptor regulates $B$ cell adhesion and immunological-synapse formation, Immunity 28 (Jan 2008), 88-99.

[32] Y. Fukui, O. Hashimoto, T. Sanui, T. Oono, H. Koga, M. Abe et al., Haematopoietic cell-specific CDM family protein DOCK 2 is essential for lymphocyte migration, Nature $\mathbf{4 1 2}$ (23 Aug 2001), 826-831.

[33] C. Nombela-Arrieta, R.A. Lacalle, M.C. Montoya, Y. Kunisaki, D. Megías, M. Marqués et al., Differential requirements for DOCK2 and phosphoinositide-3-kinase gamma during $\mathrm{T}$ and B lymphocyte homing, Immunity 21 (Sep 2004), 429-441. 


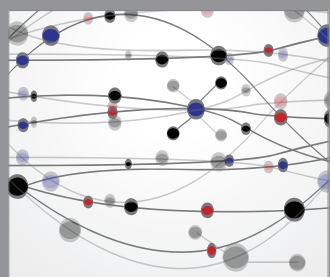

The Scientific World Journal
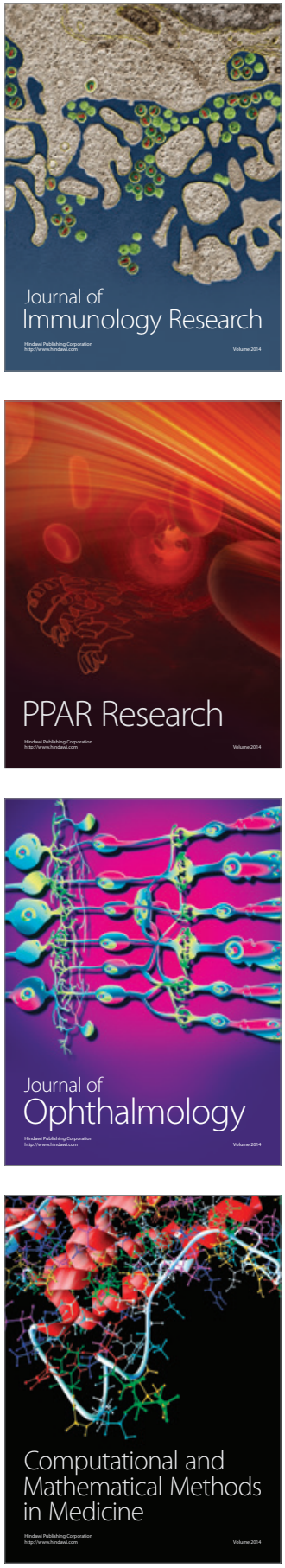

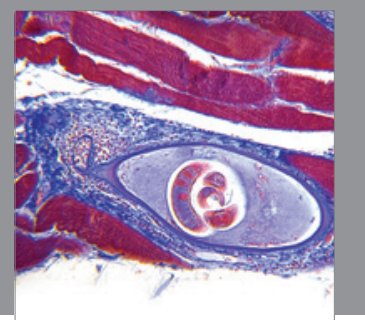

Gastroenterology

Research and Practice
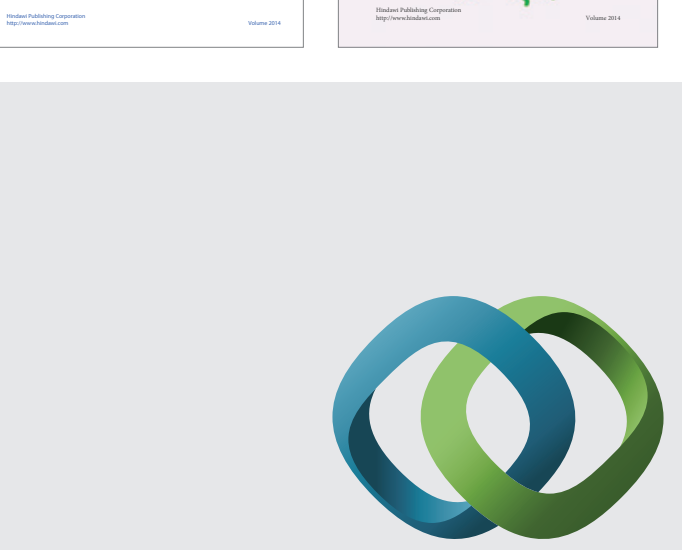

\section{Hindawi}

Submit your manuscripts at

http://www.hindawi.com
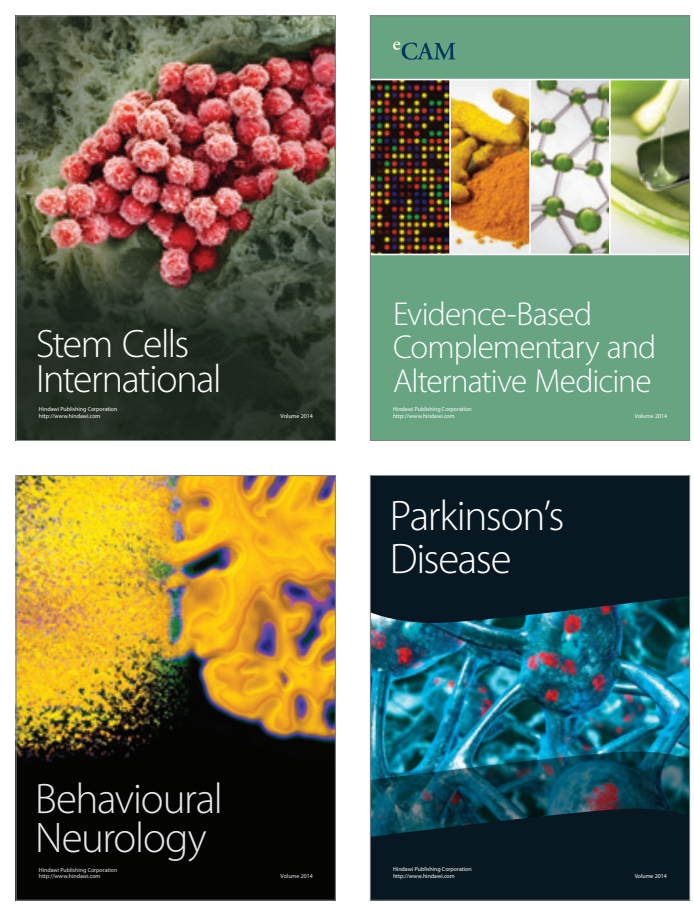

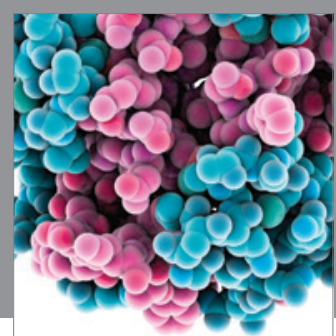

Journal of
Diabetes Research

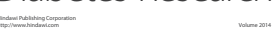

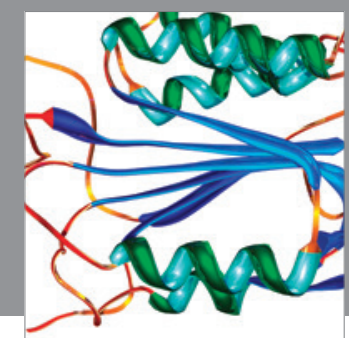

Disease Markers
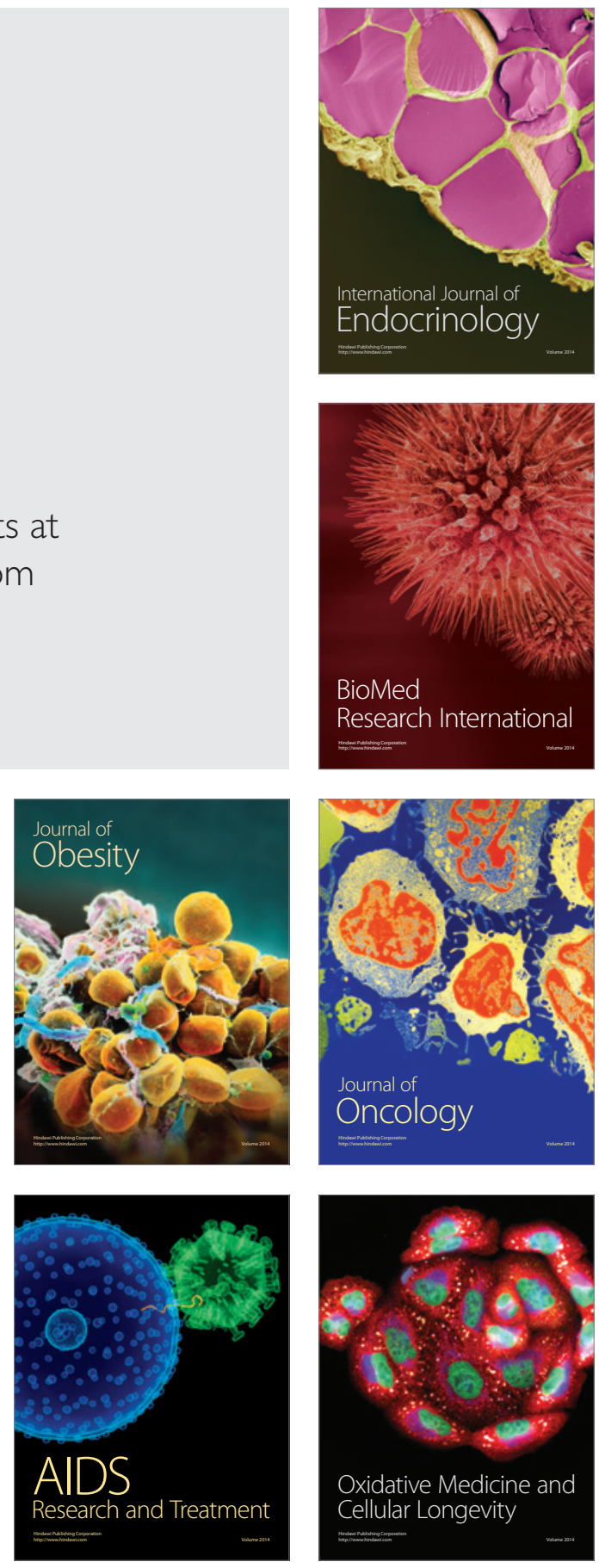